\title{
Exact Travelling Wave Solution of Fractional Space-time Zakharov-Kuznetsov Equation
}

\author{
A. H. A. Ali ${ }^{1}$, A. A. Soliman ${ }^{1 *}$, M. A. Abdou ${ }^{2}$ and M. H. Emara ${ }^{1}$ \\ 'Department of Mathematics, College of Science, University of Bisha, Bisha, 61922, PO Box 344, \\ Kingdom of Saudi Arabia; ahaali_49@yahoo.com, asoliman_99@yahoo.com \\ 2Department of Physics, College of Science, University of Bisha, Bisha 61922, PO Box 344, \\ Kingdom of Saudi Arabia; m_abdou_eg@yahoo.com
}

\begin{abstract}
Objectives: To find the new exact travelling wave solutions of fractional order space-time Zakharov- Kuznetsov (ZK) equation of ion-acoustic waves. Methods/Statistical: The fractional derivative is defined in the sense of modified Riemann- Liouville derivative to convert the fractional space-time ZZK equation to nonlinear ordinary differential equation," The two proposed techniques the $\left(\omega^{\prime} / \omega\right)$-expansion and extended $\left(\omega^{\prime} / \omega\right)$-expansion methods are employed for constructing the new exact travelling wave solutions of ZK equation. Findings/Results: The obtained new exact travelling wave solutions of ZK equation include the hyperbolic function, rational function, and trigonometric function. Application: The results reveal that the two used methods here are effective and powerful methods and might be accustomed to establish the other solutions of nonlinear fractional partial differential equations that arising in [nonlinear science."
\end{abstract}

Keywords: The $\left(\omega^{\prime} / \omega\right)$-Expansion Method, The Extended $\left(\omega^{\prime} / \omega\right)$-Expansion Method, The Modified Riemann- Liouville Derivative, Homogeneous Balance, The New Exact Travelling Wave Solutions

\section{Introduction}

The fractional calculus has a wide array namely, nonlinear optic, solid state physics, fluid flow, plasma physics, control theory, signal processing, systems identifications, biology ${ }^{1-28}$ and so on. During this paper the target of our aim by means that two proposed methods, namely and extended - expansions methods to solve the following fractional space-time in nonlinear of ion-acoustic waves arising in plasma physics as $\underline{29-31}$.

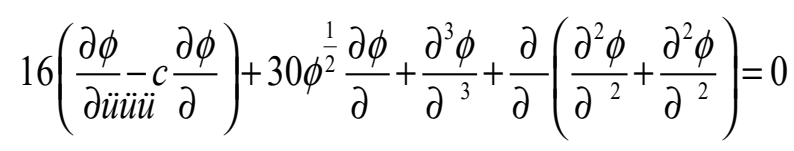

Eq.(1.1a) can be rewritten in the fractional space-time form as

$$
16\left(\frac{\partial^{\alpha} \phi}{\partial t^{\alpha}}-c \frac{\partial^{\alpha} \phi}{\partial x^{\alpha}}\right)+30 \phi^{\frac{1}{2}} \frac{\partial^{\alpha} \phi}{\partial x^{\alpha}}+\frac{\partial^{3 \alpha} \phi}{\partial x^{3 \alpha}}+\frac{\partial}{\partial x^{\alpha}}\left(\frac{\partial^{2 \alpha} \phi}{\partial y^{2 \alpha}}+\frac{\partial^{2 \alpha} \phi}{\partial z^{2 \alpha}}\right)=0
$$

where, $\mathrm{c}$ is constant, consider the fractional transform $^{26}$

$$
\phi(x, y, z, t)=G(\theta) \text {, and } \theta=\frac{\text { üiü }}{\Gamma(1+\alpha)}+\frac{\alpha}{\Gamma(1+\alpha)}+\frac{\rho^{\alpha}}{\Gamma(1+\alpha)}-\frac{\alpha}{\Gamma(1+\alpha)} \text {, }
$$

${ }^{*}$ Author for correspondence 
where, and are constants to be resolved later on. By using Eq. (1.2) into Eq. (1.1b) can be reduced to

$$
16(w-k c) G^{\prime}+30 G^{\frac{1}{2}} G^{\prime}+\left(k^{3}+k l^{2}+k \rho^{2}\right) G^{\prime \prime}=0
$$

Integrate Eq. (1.3) and chose the constant of integration to be zero, we deduce

$$
16(w-k c) G+20 G^{\frac{3}{2}}+\left(k^{3}+k l^{2}+k \rho^{2}\right) G^{\prime \prime}=0
$$

$$
\begin{aligned}
& \text { Using } G^{\frac{1}{2}}=V \text { Eq.(1.4) becomes } \\
& 16(w-k c) V^{2}+20 V^{3}+2\left(k^{3}+k l^{2}+k \rho^{2}\right)\left(\left(V^{\prime}\right)^{2}+V V^{\prime \prime}\right)=0
\end{aligned}
$$

This paper has been outlined as follows: Section 2represents the modified Riemann- Liouville derivative and it's a few properties. In sections $3 \mathrm{~A}, 3 \mathrm{~B}$ two proposed techniques are explained. In sections 4,5 the obtained solutions of the reduced Eq. (1.5) have been presented. In last section the conclusion is given.

\section{The Modified Riemann- Liouville Derivative and Some Properties}

In this section, the Jumarie's Riemann-Liouville derivative is outlined as follows ${ }^{1-2}$

$$
D_{t}^{\alpha} g(t)=\left\{\begin{array}{ll}
\frac{1}{\Gamma(1-\alpha)} \int_{0}^{t}(t-\eta)^{-\alpha-1}[g(\eta)-g(0)] d \eta, & \alpha<0 \\
\frac{1}{\Gamma(1-\alpha)} \frac{d}{d t} \int_{0}^{t}(t-\eta)^{-\alpha}[g(\eta)-g(0)] d \eta, & \alpha<1 \\
\left(g^{(m)}(t)\right)^{(\alpha-m)} & m \leq \alpha<m+1 ; m \geq 1
\end{array}\right\}
$$

With the properties:

i) $\quad D_{t}^{\alpha} t^{\gamma}=\frac{\Gamma(1+\gamma)}{\Gamma(1+\gamma-\alpha)} t^{\gamma-\alpha}, \gamma>0$,

ii) $D_{t}^{\alpha}(a g(t)+b f(t))=a D_{t}^{\alpha} g(t)+b D_{t}^{\alpha} f(t)$,

where $\mathrm{a}, \mathrm{b}$ are constants.

iii) $D_{t}^{\alpha} g(\eta)=\frac{d g}{d \eta} D_{t}^{\alpha} \eta, \eta=g(t)$,

iv) $\quad D_{t}^{\alpha} c=0, \quad c$ is constant (2.5)

v) $d^{\alpha} x(t)=\Gamma(1+\alpha) d x(t)$.

\section{Algorithms of the Two Proposed Method}

\subsection{Methodology of the -Expansion Technique}

In what follows, we explain the algorithm of the expansion method as follows:

Step 1: Assume the general fractional space-time NPDE as

$$
H\left(v, D_{t}^{\alpha} v, D_{x}^{\beta} v, D_{t t}^{2 \alpha} v, D_{x x}^{\beta} v, D_{t}^{\alpha} D_{x}^{\beta} v, \ldots .\right), 0<\alpha, \beta<1,
$$

where, is an unknown functionin two independent variables and, is a polynomial in terms of , and its derivatives and including the nonlinear terms. 
Step 2: Consider the travelling Wavetrans form as:

$$
v(x, t)=V(\eta), \quad \eta=\frac{k x^{\alpha}}{\Gamma(1+\alpha)}+\frac{c t^{\alpha}}{\Gamma(1+\alpha)} .
$$

Inserting Eq.(3.2a) into (3.1a), we obtain:

$$
Z\left(V, c V^{\prime}, V^{\prime}, c^{2} V^{\prime \prime}, c V^{\prime \prime}, V^{\prime \prime}, V V^{\prime}, \ldots\right)=0
$$

Step 3: We express the solution of ODE (3.3a) in the form:

$$
V(\eta)=\sum_{i=0}^{N} \beta_{i}\left(\frac{\omega^{\prime}}{\omega}\right)^{i}
$$

where, satisfies

$$
\omega^{\prime \prime}+\lambda \omega^{\prime}+\mu \omega=0
$$

where, $\beta_{i}, \lambda$ and $\mu$ can be specified later on, $\beta_{i} \neq 0$.

Step 4: Inserting Eq.(3.4a) into Eq. (3.3a) with Eq. (3.5a), equating the order of the same order of to be zero, we get the set of algebraic equations for $\beta_{i}, \mathrm{c}, \lambda$ and $\mu$. By solving the system of algebraic equations with the solutions of Eq. (3.5) admits to the solutions of Eq.(3.1a).

\subsection{The Extended -Expansion Technique}

In what follows, the proposed technique ${ }^{20}$ is described. For a give general partial differential equation as

$$
H\left(v, D_{t}^{\alpha} v, D_{x}^{\beta} v, D_{t t}^{2 \alpha} v, D_{x x}^{\beta} v, D_{t}^{\alpha} D_{x}^{\beta} v, \ldots .\right), 0<\alpha, \beta<1,
$$

To derive the exact solution of Eq. (3.1b), consider the fractional transformation:

$$
v \text { ü̈̈̈̈̈ }=V \quad \eta \quad \eta=\frac{k x^{\alpha}}{\Gamma(1+\alpha)}+\frac{c t^{\alpha}}{\Gamma(1+\alpha)}
$$

Substituting Eq.(3.2b) into Eq.(3.1a) transfers o

$$
Z\left(V, c V^{\prime}, V^{\prime}, c^{2} V^{\prime \prime}, c V^{\prime \prime}, V^{\prime \prime}, V V^{\prime}, \ldots\right)=0
$$

We express the solution $V(\eta)$ as finite series in the form

$$
V(\eta)=\sum_{i=0}^{N} \beta_{i}\left(\frac{\omega^{\prime}}{\omega}\right)^{i}
$$

where, $\mathrm{N}$ is the balancing number which is positive integer, it is evaluated by considering the homogeneous balance. $\omega=\omega(\eta)$ satisfies

$$
\omega \omega^{\mathrm{D}}=D \omega^{2}+E \omega \omega^{\prime}+F\left(\omega^{\prime}\right)^{2}
$$

where, D, E and F are real parameters should be determined later.

\section{Application of the $\left(\omega^{\prime} / \omega\right)-$ Expansion Technique to the ZK Equation}

Apply the $\left(\omega^{\prime} / \omega\right)$-expansion technique to extract the exact solution for ZK equation (1.5). Assume that the homogenous balancing between $V^{3}$ with $V V^{\prime \prime}$, we get $N=2$. Then the solutions of Eq. (1.5) is defined as:

$$
V(\eta)=\beta_{0}+\beta_{1}\left(\frac{\omega^{\prime}}{\omega}\right)+\beta_{2}\left(\frac{\omega^{\prime}}{\omega}\right)^{2}
$$

Satisfies Eq. (3.5a), are constants should be calculated later on.

Inserting Eq. (4.1) into Eq. (1.5) and equating the same power of $\left(\omega^{\prime} / \omega\right)$ to be zero. We get the set of algebraic equations for $\beta_{0}, \beta_{1}, \beta_{2}, k, l, w$. By solving this set of algebraic equations, we have 
$\beta_{0}=-k^{3} \mu-k l^{2} \mu-k \rho^{2} \mu, \quad \beta_{1}=-k^{3} \lambda-k l^{2} \lambda-k \rho^{2} \lambda, \quad \beta_{2}=-k^{3}-k l^{2}-k \rho^{2}$,

$w=\frac{\left(4 k \mu-k^{2} \lambda^{2}-\lambda^{2} l^{2}-\lambda^{2} \rho^{2}+4 \mu l^{2}+4 \mu \rho^{2}+4 c\right) k}{4}, k=k, l=l$

By inserting Eq. (4.2) into Eq.(4.1), admits the following new three exact solutions as follows:

Case I: If $\Delta=\lambda^{2}-4 \mu>0$, then the hyperbolic function solution is:

$$
V_{1}=-\alpha_{1} k\left(\mu+\delta_{1} \lambda+\delta_{1}^{2}\right)
$$

where,

$$
\begin{aligned}
& \alpha_{1}=k^{2}+l^{2}+\rho^{2}, c_{1}=\frac{\sqrt{\Delta}}{2} . \\
& \delta_{1}=c_{1}\left(\frac{h_{1} \sinh \left(c_{1} \eta\right)+h_{2} \cosh \left(c_{1} \eta\right)}{h_{1} \cosh \left(c_{1} \eta\right)+h_{2} \sinh \left(c_{1} \eta\right)}\right)-\frac{\lambda}{2}
\end{aligned}
$$

Case II: If $\Delta=\lambda^{2}-4 \mu<0$, then the trigonometric function solution is:

$$
V_{2}=-k \alpha_{1}\left(\mu+\lambda \delta_{2}+\delta_{2}^{2}\right)
$$

where,

$$
\begin{aligned}
& \alpha_{1}=k^{2}+l^{2}+\rho^{2}, \\
& c_{1}=\frac{\sqrt{\Delta}}{2}, c_{2}=\frac{\sqrt{-\Delta}}{2}, \\
& \delta_{2}=c_{2}\left(\frac{-h_{1} \sin \left(c_{1} \eta\right)+h_{2} \cos \left(c_{1} \eta\right)}{h_{1} \cos \left(c_{1} \eta\right)+h_{2} \sin \left(c_{1} \eta\right)}\right)-\frac{\lambda}{2}
\end{aligned}
$$

Case III: If $\Delta=\lambda^{2}-4 \mu=0$, then the rational function solution is:

$$
V_{3}=-k \alpha_{1}\left(\mu+\lambda \delta_{3}+\delta_{3}^{2}\right)
$$

where,

$$
\alpha_{1}=k^{2}+l^{2}+\rho^{2}
$$

$$
\delta_{3}=\left(\frac{k_{2}}{k_{1}+k_{2} \eta}\right)-\frac{\lambda}{2}
$$

$$
G^{\frac{1}{2}}(\eta)=V(\eta), \eta=\frac{k x^{\alpha}}{\Gamma(1+\alpha)}+\frac{l y^{\alpha}}{\Gamma(1+\alpha)}+\frac{\rho z^{\alpha}}{\Gamma(1+\alpha)}-\frac{w t^{\alpha}}{\Gamma(1+\alpha)}
$$

\section{Application of Extended - Expansion Method to the ZK Equation}

Here, to solve Eq.(1.5) via the extended -expansion method. Consider the balancing with, wed educe $N=2$.Therefore, the solution of Eq.(1.5) becomes

$$
V(\eta)=\beta_{0}+\beta_{1}\left(\frac{\omega^{\prime}}{\omega}\right)+\beta_{2}\left(\frac{\omega^{\prime}}{\omega}\right)^{2}
$$

Substituting Eq. (5.1) into Eq.(1.5), we obtain the values of $\beta_{0}, \beta_{1}, \beta_{2}, k, l, w$

$$
\begin{aligned}
& \beta_{0}=-k^{3} D F-k D l^{2} F-k D \rho^{2} F+k l^{2} D+k D \rho^{2}+k^{3} D, \\
& \beta_{1}=k l^{2} E+k \rho^{2} E+k^{3} E-k^{3} E F-k l^{2} E F-k \rho^{2} E F, \\
& \beta_{2}=-k^{3} F^{2}-k l^{2} F^{2}-k \rho^{2} F^{2}+2 k^{3} F-k^{3}+2 k l^{2} F-k l^{2}+2 k \rho^{2} F-k \rho^{2} \\
& w=\frac{\left(4 k^{2} D F+4 D \rho^{2} F+4 D l^{2} F-k^{2} E^{2}-4 k^{2} D-4 l^{2} D-4 \rho^{2} D-l^{2} E^{2}-\rho^{2} E^{2}+4 c\right) k}{4}, \\
& k=k, l=l, c=c, D=D
\end{aligned}
$$

By inserting Eq. (5.2) into Eq. (5.1), we conclude that a new four exact solutions to Eq.(1.5) as follows: 
Case I: If $E \neq 0$ and $\Delta=E^{2}+4 D-4 D F \geq 0$, then the solution is:

$$
V_{1}=-k \alpha_{1}\left(-D(F-1)+E^{2} \gamma_{1}+E^{3} \gamma_{1}^{2}\right)
$$

$$
\begin{aligned}
& \text { where, } \\
& \alpha_{1}=k^{2}+l^{2}+\rho^{2}, c_{1}=\frac{\sqrt{\Delta}}{2} \\
& \gamma_{1}=\frac{1}{2}+c_{1}\left(\frac{h_{1} \exp \left(c_{1} \eta\right)+h_{2} \exp \left(-c_{1} \eta\right)}{h_{1} \exp \left(c_{1} \eta\right)-h_{2} \exp \left(-c_{1} \eta\right)}\right)
\end{aligned}
$$

Case II: If $E \neq 0$ and $\Delta=E^{2}+4 D-4 D F<0$, then the trigonometric function solution is:

$$
V_{2}=-k D \alpha_{1}\left(-D(F-1)+k E^{2} \alpha_{1} \gamma_{2}+\gamma_{2}^{2} E^{3}\right)
$$

where,

$$
\begin{gathered}
\alpha_{1}=k^{2}+l^{2}+\rho^{2}, c_{1}=\frac{\sqrt{\Delta}}{2}, c_{2}=\frac{\sqrt{-\Delta}}{2}, \\
\gamma_{2}=\frac{1}{2}+c_{2}\left(\frac{i h_{1} \cos \left(c_{2} \eta\right)-h_{2} \sin \left(c_{2} \eta\right)}{\left.\left.i h_{1} \sin \left(c_{1} \eta\right)\right)+h_{2} \cos \left(c_{1} \eta\right)\right)}\right)
\end{gathered}
$$

Case III: If $E=0$ and $\Delta=D(1-F) \geq 0$, then the trigonometric function solution is:

$$
V_{3}=-k \alpha_{1}\left(D(F-1)-\gamma_{3}^{2}\right)
$$

where,

$$
\begin{aligned}
& \alpha_{1}=k^{2}+l^{2}+\rho^{2}, c_{3}=\sqrt{\Delta}, \\
& \gamma_{3}=c_{3}\left(\frac{h_{1} \cos \left(c_{3} \eta\right)+h_{2} \sin \left(c_{3} \eta\right)}{h_{1} \sin \left(c_{3} \eta\right)-h_{2} \cos \left(c_{3} \eta\right)}\right)
\end{aligned}
$$

Case IV: If $E=0$ and $\Delta=D(1-F)<0$, then the hyperbolic function solution is:

$$
V_{4}=-k \alpha\left(D(F-1)-\gamma_{4}^{2}\right)
$$

where,

$$
\begin{aligned}
& \alpha_{1}=k^{2}+l^{2}+\rho^{2}, c_{4}=\sqrt{-\Delta} \\
& \gamma_{4}=c_{4}\left(\frac{i h_{1} \cosh \left(c_{4} \eta\right)-h_{2} \sinh \left(c_{4} \eta\right)}{i h_{1} \sinh \left(c_{4} \eta\right)-h_{2} \cosh \left(c_{4} \eta\right)}\right)
\end{aligned}
$$

where,

$G^{\frac{1}{2}}(\eta)=V(\eta), \eta=\frac{k x^{\alpha}}{\Gamma(1+\alpha)}+\frac{l y^{\alpha}}{\Gamma(1+\alpha)}+\frac{\rho z^{\alpha}}{\Gamma(1+\alpha)}-\frac{w t^{\alpha}}{\Gamma(1+\alpha)}$

\section{Conclusion}

In this study, the-expansion and the extended-expansion methods have been applied for obtaining the new exactsolutions of ZK equation of fractional order arising in physics. The obtained solutions can be expressed as trigonometric, hyperbolic and rational functions. The fractional transformation is used to convert the partial differential equation into the ordinary differential equation. The solutions reported here have not been published elsewhere.

Finally, the two planned methods are direct, concise, elementary, and effective and might be used for solving other nonlinear partial differential equation fractional order. This is often our duty in the future.

\section{Acknowledgement}

This Paper has been sponsored by the Research Sponsorship Program, University of Bisha, Kingdom of Saudi Arabia: grand Number (UB-016-1438). 


\section{References}

1. Jumarie G. Modified Riemann-Liouville derivative and Fractional Taylor series of non-differentiable functions further results. Computers and Mathematics with Applications. 2006; 51(9-10):1367-76.

2. Jumarie G. Table of some basic fractional calculus formula derived from a Modified Riemann-Liouville derivative for non-differentiable functions. Applied Mathematics Letters. 2009; 22(3):-378-85.

3. Kumar S. A numerical study for solution of time fractional nonlinear shallow-water equation in oceans. Zeitschrift für Naturforschung A Journal of Physical Sciences. 2003; 68(89):547-53.

4. Kumar S, Kocak H, Yildirim A. a fractional model of gas dynamics equation and its approximate solution by using Laplace Transform. Zeitschrift für Naturforschung A Journal of Physical Sciences. 2012; 67(6-7):389-96.

5. Agarwal RP, Benchohra M, Hamani S. A survey on existence results for boundary value problems of nonlinear fractional differential equations and inclusions. Acta Applicandae Mathematicae. 2010; 109(3):973-1033. https://doi.org/10.1007/s10440-008-9356-6.

6. Shou DH, He JH. Beyond Adomain Method: The variational iteration method for solving heat like and wave-like equation with variable coefficients. Physics Letters A. 2008; 372(3):233-7. https://doi.org/10.1016/j.physleta.2007.07.011.

7. Kumar D, Singh J, Kumar S. Analytical modelling for fractional multi-dimensional diffusion equations by using Laplace Transform. Communications in Numerical Analysis. 2015; 1(2015):16-29. https://doi.org/10.5899/2015/cna-00220.

8. Baleanu D. About fractional quantization and fractional variational principles. Communications in Nonlinear Science and Numerical Simulation. 2009; 14(6):2520-3. https://doi.org/10.1016/j.cnsns.2008.10.002.

9. Gepreel KA, Omran S. Exact solutions for nonlinear partial fractional differential equations. Chinese Physics B. 2012; 21(11):110204.

10. Ray SS, Bera RK. An approximate solution of a nonlinear fractional differential equation by Adomians Decomposition Method. Applied Mathematics and Computation. 2005; 167(1):561-71.

11. Zhang S, Zhang HQ. Fractional Sub-equation method and its applications to nonlinear fractional PDEs. Physics Letters A. 2011; 375(7):-1069-73.

12. Zhao J, Tang B, Kumar S, Hou Y. The extended fractional sub-equation -method for nonlinear fractional differential equations. Mathematical Problems in Engineering; 2012. p. $1-11$.
13. Tong B, He Y, Wei L, Zhang X. A generalized fractional sub-equation method for fractional differential equations with variable coefficients. Physics Letters A. 2012; 376(3839):2588-90.

14. Guo S, Mei L, Li Y, Sun Y. The improved fractional subequation method and its applications to the space-time fractional differential equations in fluid mechanics. Physics Letters A. 2012; 376(4):407-11.

15. Bin L. Backlund transformation of fractional Riccati Equation and its applications to nonlinear fractional partial differential equations. Physics Letters A. 2012; 376(28-29):2045-8.

16. Elgarayhi AA, Abdou MA, Attia AT. New exact solutions for space- time fractal order on the ion Acoustic Waves in electron-positron-ion plasma. Nonlinear Science Letters A Mathematics, Physics and Mechanics. 2014; 5:35-44.

17. Abdou MA, Elgarayhi AA, El-Shewy E. Fractional complex transform for space-time fractional nonlinear differential equations arising in plasma physics. Nonlinear Science Letters A Mathematics, Physics and Mechanics. 2014; 5(1):31-4.

18. Abdou MA, Elhanbaly A. New application of the fractional sub-equation method. Nonlinear Science Letters A: Mathematics, Physics and Mechanics. 2015; 6(1):1018 .

19. Abdou MA, Yildirim A. Approximate analytical solution to time fractional nonlinear evolution equations. International Journal of Numerical Methods for Heat and Fluid Flow. 2015; 22(7):829-38.

20. Abdou MA. A new analytical method for space-time fractional nonlinear differential equations arising in plasma physics. Journal of Ocean Engineering and Science. 2017; 2(4):1-5.

21. Abdou MA, Elhanbaly A, Attia MT. New exact solutions of the space-time fractional nonlinear transport equation in porous media. Nonlinear Science Letter A. 2016; 7(3):8695.

22. Alam MN, Hafez MG, Akbar MA, Roshid HO. Exact solutions to the $(2+1)$ Dimensional Boussinesq Equation via Exp-Expansion Method. Journal of Scientific Research. 2015; 7(3):1-10.

23. Kaplan M, Bekir A. A novel analytical method for time fractional differential equations. Optik. 2016; 127(20):8209-14. https://doi.org/10.1016/j.ijleo.2016.05.152.

24. Roshid HO, Md. Rahman A. The $\exp (-\Phi(\eta))$ expansion method with application in the $(1+1)$ dimensional classical Boussinesq equations. Results in Physics. 2014; 4:150-5.

25. Feng Q. Exact solutions for fractional partial differential equations by a new generalized fractional sub- 
equation method. Wseas Transactions on Mathematics. 2016; 15:1-8.

26. He JH, Li ZB. Fractional complex transform for fractional differential equations. Math. Mathematical and Computational Applications. 2010; 15(5):970-3.

27. Meng F, Feng Q. A new fractional sub-equation method and its applications for space-time fractional partial differential equations. Journal of Applied Mathematics; 2013. p. 1-10.

28. Bin Z, Chuanbao W. Exact solutions for fractional partial differential- equations by a New Fractional sub-equation method. Advances in Difference Equations; 2013. p. 199.

29. Ray SS, Sahoo S. New exact solutions of fractional ZakharovKuznetsov and Modified Zakharov-Kuznetsov equations using fractional sub-equation method. Communications in Theoretical Physics. 2015; 63(1):25-30. https://doi. org/10.1088/0253-6102/63/1/05.

30. Seadawy AR. Three-dimensional nonlinear Modified Zakharov- -Kuznetsov equation of ion-acoustic waves in a magnetized plasma. -Computers and Mathematics with Applications. 2016; 71(1):201-12. https://doi.org/10.1016/j. camwa.2015.11.006.

31. Yildırım A, Gülkanat Y. Analytical approach to fractional Zakharov-Kuznetsov equations by He's Homotopy Perturbation Method. Communications in Theoretical Physics. 2010; 53(6):1005. https://doi.org/10.1088/02536102/53/6/02. 